\title{
DESENVOLVIMENTO DE UM EQUIPAMENTO PARA MEDIÇÃO DA TEMPERATURA CORPÓREA DE DEFICENTES VISUAIS
}

\author{
A. O. Andrade**, C. J. C. Trindade*, M. M. Soares*, R. H. B. S. Silva* e T. L. Maganha* \\ * Faculdade de Engenharia Elétrica, Universidade Federal de Uberlândia, Uberlândia, Brasil \\ ** Núcleo de Inovação e Avaliação Tecnológica em Saúde, Universidade Federal de Uberlândia, \\ Uberlândia, Brasil \\ e-mail: chrystian.ctrindade@outlook.com
}

\begin{abstract}
Resumo: A interface entre homem-máquina pode ter várias aplicações, inclusive na área de Tecnologias Assitivas (TA), pois atualmente, com os avanços da tecnologia, muitos estudos são feitos para melhorar a qualidade de vida das pessoas e, principalmente, dos deficientes, seja qual for esta deficiência. Vistas todas as dificuldades enfrentadas pelos deficientes visuais, foi analisado e implementado este projeto na área de TA que consiste em um medidor de temperatura corpórea (mais conhecido como termômetro) para o auxílio a tais deficientes. Portanto, o equipamento permite que estas pessoas possuam uma vida mais independente, podendo ter um feedback auditivo.
\end{abstract}

Palavras-chave: tecnologia assistiva, deficientes visuais, termômetro.

\begin{abstract}
The man-machine interface can have multiple applications, including the area of Assistives Technologies (AT), as currently, with advances in technology, many studies are made to improve the quality of life of the people and, especially, the disabled, whatever this deficiency is. Owing to all the difficulties faced by the visually impaired, was analyzed and implemented this project in the area of AT that consists of a body temperature (thermometer) for assistance to them. Therefore, the equipment allows these people to have a more independent life, with the auditory feedback.
\end{abstract}

Keywords: assistive technology, visually impaired, thermometer.

\section{Introdução}

Entende-se por Tecnologia Assistiva (TA) como recursos que auxiliem na potencialização das habilidades de pessoas com algum tipo de deficiência física, ou seja, tem como finalidade o desenvolvimento individual de pessoas com algum tipo de limitação [1].

Os cegos contam, atualmente, com variados recursos que auxiliam em seu processo de apreensão e aprendizagem dos principais conteúdos do conhecimento: materiais adaptados, Livro Falado, Sistema de Leitura Ampliada, Thermoform. Além disso, nos microcomputadores acessíveis, existe o Terminal
Braille, Impressora Braille, Scanner de Mesa e, especialmente, os Sintetizadores de Voz, com destaque para o Sistema Operacional Dosvox, cuja grande aceitação pelos usuários, tanto em nível domiciliar quanto em nível escolar, tornou-o um dos mais utilizados em todo o país [2].

Vive-se um momento em que a tecnologia é altamente valorizada. O mundo atual funciona a partir de tecnologias cada vez mais sofisticadas e a crença na sua eficácia é significativa. Sem dúvida, os recursos técnicos podem facilitar a vida moderna do homem. No entanto, é importante estar atento ao fato de que ela pode não contemplar a totalidade das necessidades das pessoas [3].

A Tecnologia Assistiva envolve tanto o objeto, ou seja, a tecnologia concreta (o equipamento ou instrumento), quanto o conhecimento requerido no processo de avaliação, criação, escolha e prescrição, isto é, a tecnologia teórica. Tem como áreas de aplicação: adaptações para atividades da vida diária; sistemas de comunicação alternativa; dispositivos para utilização de computadores; unidades de controle ambiental; adaptações estruturais em ambientes domésticos, profissionais ou público; adequação da postura sentada; adaptações para déficits visuais e auditivos; equipamentos para mobilidade; adaptações em veículos [3].

O cego e o deficiente visual severo são estimulados a serem independentes nas suas atividades de vida diária. Quando frequentam escola especial são habilitados na arte de preparar seus alimentos, cuidar de suas roupas e de sua casa, e se têm filhos devem estar preparados para cuidá-los. A habilitação para a vida diária do cego instrumentaliza-o para executar estas tarefas com segurança, sem dúvida, quando não tem esta oportunidade de treinamento, está mais exposto a riscos [4].

A partir disto, o presente estudo objetivou a implementação de um projeto de que consiste na medição da temperatura corpórea destinado à deficientes visuais, que possui feedback auditivo, introduzindo este benefício da TA na vida dessas pessoas, deixando-as mais independentes com eventos cotidianos, mostrando que a TA deve ser cada dia mais introduzida na vida dos deficientes. 


\section{Materiais e métodos}

Trata-se de um estudo de avaliação e implementação de um equipamento em Tecnologia Assistiva, que consiste num medidor da temperatura corpórea (mais conhecido como termômetro) para deficientes visuais. $\mathrm{O}$ equipamento consiste em um hardware que foi projetado segundo especificações técnicas dos componentes que o compõe, sendo este controlado por um microcontrolador. A temperatura é captada por um sensor, que é um sinal analógico, lido pelo microcontrolador. A configuração de montagem se apresenta na Figura 1. O software manipula esses dados adquiridos e executa segundo um comando estabelecido. Assim, um feedback auditivo é executado, informando ao deficiente se eles está com febre ou não.

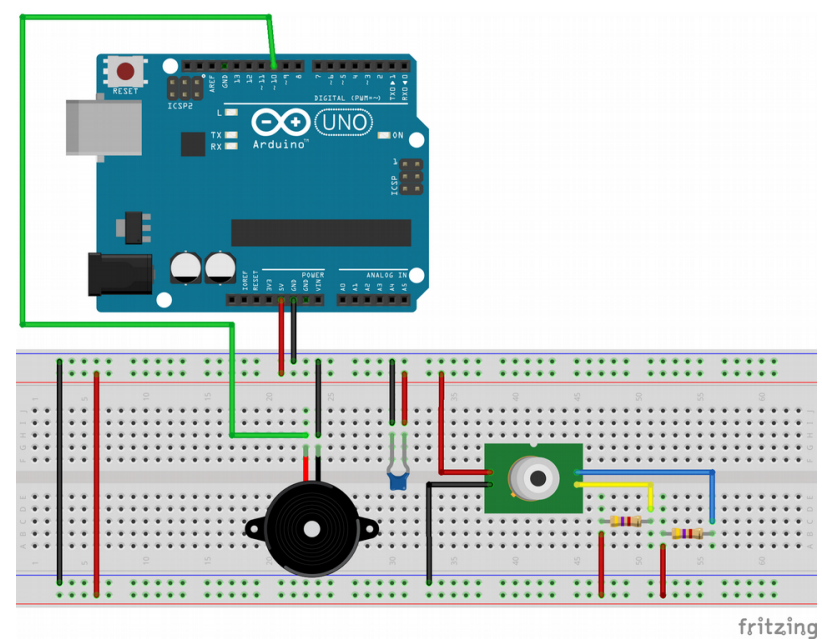

Figura 1: Configuração de montagem do hardware.

Como documentação do projeto, foi feita uma pesquisa bibliográfica na base de dados Scielo e Google Acadêmico, com a finalidade de obter artigos científicos. Com os resultados obtidos com esta pesquisa foi possível deter maiores conhecimentos acerca do tema "Tecnologia Assistiva", além de informações sobre a acessibilidade e a inserção do deficiente auditivo na sociedade não adaptada à ele.

A fase inicial de execução do projeto foi de maiores especificações sobre os componentes que foram utilizados na montagem do hardware. Posteriormente, uma pesquisa foi feita sobre as bibliotecas e códigos necessários para o software, que foi programado na plataforma de programação de linguagem do Arduino.

Arduino - plataforma de prototipagem eletrônica open-source que se baseia em hardware e software de uso acessível e fácil. O microcontrolador da placa é programado com a linguagem de programação Arduino, baseada na linguagem Wiring, e o ambiente de desenvolvimento Arduino, baseado no ambiente Processing.

Para fins de verificação visual, caso seja necessário, consegue-se plotar as informações captadas na plataforma de desenvolvimento do software, que, no caso, é a temperatura medida. Essa plotagem acontece com uma frequência estabelecida no código de manipulação dos dados.

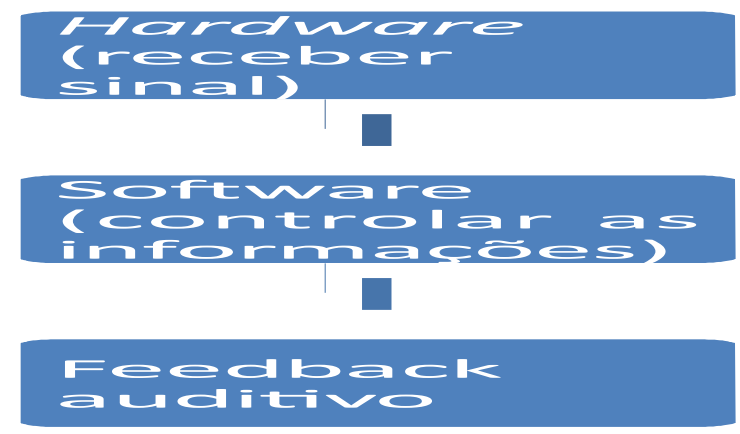

Figura 2: Diagrama de blocos do funcionamento.

Para montagem do software, foi necessária a instalação da biblioteca do Arduino "i2master.h”.

\section{Resultados}

Os resultados do projeto foram satisfatórios, visto que cumpre seu objetivo de dar um feedback auditivo ao usuário. O feedback se dá através do componente buzzer, que emite som quando o sensor MLX 90614 processa temperaturas acima de $37.8{ }^{\circ} \mathrm{C}$, indicativo de febre no usuário. Para que o projeto pare de emitir som basta distanciar o sensor de temperatura do corpo e ele passe a processar somente os valores de temperatura ambiente. A montagem final do hardware segue abaixo. Como teste, foi utilizado o sistema em dos integrantes do grupo que, com os olhos vendados, conseguiu saber, aproximadamente, qual era sua temperatura vista a frequência com que o buzzer disparou o som. 


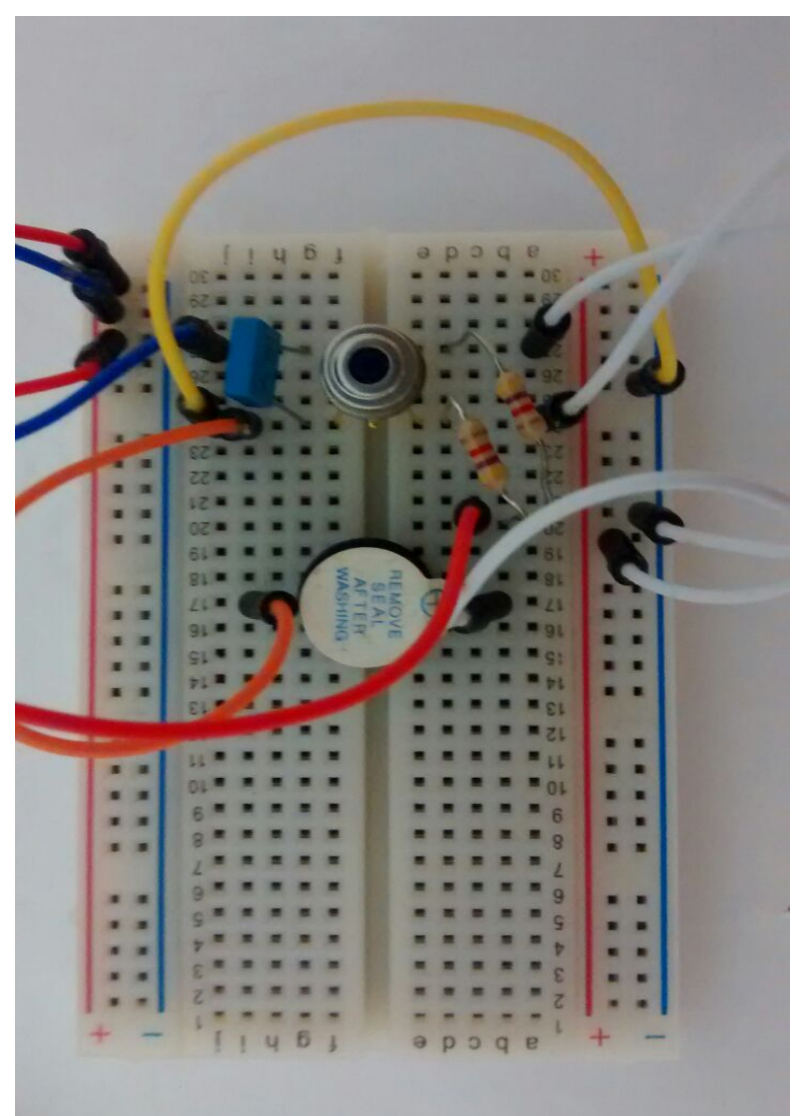

Figura 3: Montagem do hardware.

\section{Discussão}

Este projeto foi proposto como critério de avaliação da disciplina Interface-Homem Máquina, componente curricular do curso de Engenharia Biomédica da UFU.

O sensor não teve uma resposta muito precisa, dando uma variação média de dois graus Celsius ao medir a temperatura corporal.

Uma possível melhoria para o projeto seria montar o circuito desenvolvido numa placa impressa para diminuir interferências e perdas de sinal. A utilização de um outro sensor com uma melhor resolução seria uma outra alternativa.

Foi possível desenvolver o projeto, satisfazendo os objetivos propostos. Visto que é uma tecnologia que pode ser implantada, estudos futuros podem ser continuados para uma melhora em seu desenvolvimento.

\section{Conclusão}

A plataforma Arduino vem ganhando cada vez mais notoriedade e usabilidade e, devido a sua grande utilização, dispões gratuitamente de muito material de forma eletrônica para pesquisa e solução de dúvidas, além de todos os componentes eletrônicos terem seu Datasheet contendo todas as informações a seu respeito.

Logo, as informações estão facilmente dispostas para solucionar problemas que para pessoas sem alguma deficiência se mostra como simples e sem relevância mas que são de grande importância para o usuário que se quer atingir com a determinada TA.

A satisfação do grupo de desenvolvimento foi grande visto os resultados obtidos. Algumas melhorias podem ser implementadas, como discutido neste artigo, para um melhor aproveitamento do usuário.

\section{Agradecimentos}

Agradecemos ao Professor Adriano Oliveira Andrade da Faculdade de Engenharia Elétrica da Universidade Federal de Uberlândia, pela oportunidade de aprendizado e apoio durante todo o desenvolvimento do projeto.

\section{Referências}

[1] WANDERLEY, Luana Duarte et al. Desenvolvimento de tecnologia assistiva para $\mathrm{o}$ deficiente visual: utilização do preservativo masculino. 2013.

[2] CERQUEIRA, Jonir Bechara; FERREIRA, Elise de Melo Borba. Recursos didáticos na educação especial. Revista Benjamin Constant, v. 5, p. 24-29, 1996. Neuman MR. Biopotential amplifiers. In: J.G. Webster, editor. Medical Instrumentation. New York: John Wiley and Sons; 1995. p. 227-88.

[3] ROCHA, Eucenir Fredini; DO CARMO CASTIGLIONI, Maria. Reflexões sobre recursos tecnológicos: ajudas técnicas, tecnologia assistiva, tecnologia de assistência e tecnologia de apoio. Revista de Terapia Ocupacional da Universidade de São Paulo, v. 16, n. 3, p. 97-104, 2005.

[4]PAGLIUCA, Lorita Marlena Freitag et al. Desenvolvendo tecnologia para prevenção e tratamento de emergências domésticas para cegos. Revista Brasileira de Enfermagem, v. 49, n. 1, p. 83104, 1996.Onusic DM. Construção de um dispositivo eletrônico para determinação de camada semi-redutora em feixe de raios-X diagnóstico [dissertação]. Campinas: Universidade Estadual de Campinas; 2005. 